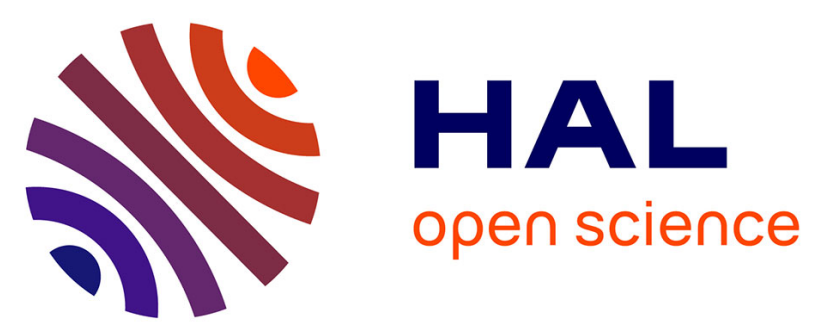

\title{
Diffusion of interstitials in metallic systems, illustration of a complex study case: aluminum
}

\author{
Matthieu David, Damien Connétable
}

\section{To cite this version:}

Matthieu David, Damien Connétable. Diffusion of interstitials in metallic systems, illustration of a complex study case: aluminum. Journal of Physics: Condensed Matter, 2017, 29 (45), 10.1088/1361$648 \mathrm{X} / \mathrm{aa} 8 \mathrm{e} 5 \mathrm{e}$. hal-01687188

\section{HAL Id: hal-01687188 \\ https://hal.science/hal-01687188}

Submitted on 18 Jan 2018

HAL is a multi-disciplinary open access archive for the deposit and dissemination of scientific research documents, whether they are published or not. The documents may come from teaching and research institutions in France or abroad, or from public or private research centers.
L'archive ouverte pluridisciplinaire HAL, est destinée au dépôt et à la diffusion de documents scientifiques de niveau recherche, publiés ou non, émanant des établissements d'enseignement et de recherche français ou étrangers, des laboratoires publics ou privés. 


\section{Open Archive TOULOUSE Archive Ouverte (OATAO)}

OATAO is an open access repository that collects the work of Toulouse researchers and makes it freely available over the web where possible.

This is an author-deposited version published in : http://oatao.univ-toulouse.fr/ Eprints ID : 19358

To link to this article :

URL : http://dx.doi.org/10.1088/1361-648X/aa8e5e

\section{To cite this version :}

David, Matthieu and Connétable, Damien Diffusion of interstitials in metallic systems, illustration of a complex study case: aluminum. (2017) Journal of Physics : Condensed Matter, vol. 29 ( $\left.{ }^{\circ} 45\right)$. pp. 1-10. ISSN 0953-8984

Any correspondence concerning this service should be sent to the repository administrator: staff-oatao@ listes-diff.inp-toulouse.fr 


\title{
Diffusion of interstitials in metallic systems, illustration of a complex study case: aluminum
}

\author{
Matthieu David and Damien Connétable \\ CIRIMAT UMR 5085, CNRS-INP-UPS, ENSIACET 4, allée Émile Monso, BP 44362, F-31030 Toulouse \\ Cedex 4, France \\ E-mail: matthieu.david@ensiacet.fr and damien.connetable@ensiacet.fr
}

\begin{abstract}
While diffusion mechanisms of interstitial elements in fcc systems are generally well-known, especially in the case of $\mathrm{H}$ atoms, we show in this work that even in the case of a simple metallic system (aluminum), the diffusion of interstitials exhibits a wide variety of paths and mechanisms that depend on the specie. We used an approach based on first-principles calculations associated with kinetic Monte-Carlo simulations and a multi-state diffusion formalism to compute the diffusion coefficients of five interstitial elements: hydrogen, boron, carbon, nitrogen and oxygen. For instance, at the atomic scale, whilst we find that $\mathrm{C}$ atoms prefer to be located in octahedral sites (labeled $o$ ) rather than in tetrahedral positions (labeled $t$ ), we find one additional stable position in the lattice $(M)$. The diffusion through these three stable positions are thus studied in detail. In the case of B atoms, for which the tetrahedral site is found unstable, the diffusion path is between $o-o$ sites. Similarly, in the case of oxygen, $t$ positions are found to be the only stable positions ( $o$ are unstable) and the path of migration, along $t-t$ direction, is found through a twice degenerated asymmetric transition state. In the case of $\mathrm{H}$ and $\mathrm{N}$ atoms for which $t$ and $o$ sites are stable, we explain why the only path is along the $t$ - $o$ direction. Finally, we discuss explicit formulas to compute coefficients of diffusion of interstitials in fcc structures.
\end{abstract}

Keywords: DFT, aluminium, diffusion, interstitials

\section{Introduction}

Diffusion in metallic compounds has been extensively studied, especially for substituted elements [1, 2]. Except for $\mathrm{H}$ atoms, the case of interstitials is often omitted. Yet in many cases, interstitials are involved in critical damaging modes of metallic systems. They interact with lattice defects (dislocations, vacancies, grain boundaries, etc) and can thus alter bulk properties. In the case of fcc systems, oxygen, carbon and hydrogen are known to lead to the $\mathrm{H}$ embrittlement of the system (corrosion cracking and corrosion fatigue) [3], to metal dusting $[4,5]$ or to internal oxidation [6]. In macroscopic models, knowledge of interstitials solubility and diffusion coefficients are one of the main information required.

In main metals, the diffusion of interstitial elements at ambient or high temperature is governed either by a direct mechanism of diffusion between stable sites [7-10] (generally tetra- and octahedral positions), or by a mechanism of traps (vacancies [11, 12], dislocation, interfaces). Regarding interstitial diffusion in fcc systems, since the stable interstitial positions in fcc systems are either the tetrahedral $(t)[7,13]$ or the octahedral $(o)$ sites $[14,15]$, the main path of diffusion is found to be between $o-t$ sites. For instance, no additional stable position, apart from $t$ and $o$ sites, was found neither in 


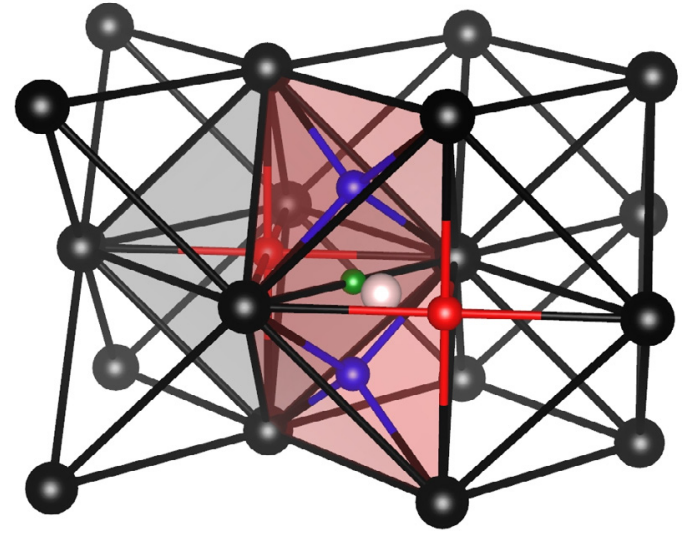

Figure 1. Schematic representation of an fcc structure (black balls), Blue and red balls respectively depict the tetrahedral and octahedral positions. We added the position of the $M$ site (green ball) and the position of the oxygen transition state (white ball).

nickel $[9,14]$ nor in palladium $[15,16]$ nor in any other fcc metals studied [17]. Due to the specific geometrical configuration of fcc structures, the transition state between two tetrahedral or two octahedral positions are exactly the same: the $M$ position, see figure 1 . As $o$ and $t$ are generally stable sites, the $M$ position thus exhibits two imaginary frequencies. In the case of nickel for instance, this was clearly observed for many elements $[9,14]$. This configuration should therefore not be seen as a classical transition state as the defined by the transition state theory. Furthermore, in early works, only $\mathrm{H}$ diffusion was clearly discussed and the explicit formula was given for this case only [9]. The aim of this manuscript is to fill this gap by presenting clear formulas of diffusion coefficients that could be applied to other metals.

Moreover, there is an evidence gap in the literature regarding atomistic diffusion mechanisms of interstitial species in aluminum. Even though many experimental works have studied the $\mathrm{H}$ diffusion (see [18] and references therein), no diffusion coefficient nor any internal friction measurement in fcc-Al have been reported in literature for other interstitial elements (C, N, O and B). In other metals however, these data exist (nickel [19-21], iron [22], etc). This lack of data can probably be explained by the low melting point of aluminum $(960 \mathrm{~K})$ and, the slow diffusivity and low solubility of interstitials.

Alike, except for $\mathrm{H}$ atoms (Wolverton et al [7]), there are few theoretical studies. For instance, Dai et al [23] only studied the relative stability of interstitial elements (from an energetic point of view) regardless if the sites were stable or not, i.e. if they are in a local minimum or not.

In this work we reinterpret density functional theory (DFT) results on interstitial stability in the light of new data and then we analyze their diffusion paths. We put an emphasis on the following interstitial elements: hydrogen, boron, carbon, nitrogen and oxygen. The study of these elements shows original paths and mechanisms at the atomic scale. DFT simulations were used to first study the stability of different configurations and then compute migration energies and frequencies of the transition states. These DFT values are then used in kinetic Monte Carlo simulations (kMC) to compute each element's macroscopic diffusion coefficient and
Table 1. Formation energies $\left(H_{f}\right.$, in $\left.\mathrm{eV}\right)$, zero-point energies ( $Z P E$, in $\mathrm{meV}$ ), volumes of Voronoï $\left(\mathcal{V}_{\text {oro }}\right.$, in $\left.\AA^{3}\right)$ and formation $\left(\Omega_{f}\right.$, in $\AA^{3}$ ) of the interstitial elements. $\delta \mathcal{V}_{\text {oro }}$ is the difference of Voronoï volume between filled and empty sites $\left(8.2,8.7\right.$ and $8.7 \AA^{-3}$ for $o, t$ and $M$ sites respectively). 'us' means unstable.

\begin{tabular}{lllllrlr}
\hline $\mathrm{X}$ & Site & $H_{f}[X]$ & $\Delta H$ & $\mathrm{ZPE}$ & $\mathcal{V}_{\text {oro }}$ & $\delta \mathcal{V}_{\text {oro }}$ & $\Omega_{f}$ \\
\hline $\mathrm{H}$ & tetra & $\mathbf{0 . 7 1 7}$ & 0 & +13 & 10.3 & 1.6 & 4.8 \\
& octa & 0.823 & 0.106 & -68 & 8.7 & 0.5 & 3.0 \\
$\mathrm{~B}$ & tetra & 2.413 & 1.184 & us & 12.7 & 4.0 & 18.8 \\
& octa & $\mathbf{1 . 2 2 9}$ & 0 & -85 & 9.8 & 1.6 & 11.1 \\
& $M$ & 1.979 & 0.750 & us & 10.6 & 2.0 & 16.6 \\
$\mathrm{C}$ & tetra & 1.691 & 0.369 & -121 & 11.1 & 2.4 & 14.5 \\
& octa & $\mathbf{1 . 3 2 2}$ & 0 & -130 & 9.0 & 0.8 & 6.4 \\
& $M$ & 1.902 & 0.580 & -146 & 9.3 & 0.7 & 12.3 \\
$\mathrm{~N}$ & tetra & $\mathbf{- 0 . 9 0 4}$ & 0 & +1 & 10.4 & 1.7 & 10.6 \\
& octa & -0.056 & 0.850 & -57 & 8.8 & 0.6 & 4.6 \\
$\mathrm{O}$ & tetra & $\mathbf{- 3 . 4 7 8}$ & 0 & +24 & 10.7 & 2.0 & 8.8 \\
& octa & -1.992 & 1.486 & us & 9.4 & 1.2 & 6.9 \\
\hline
\end{tabular}

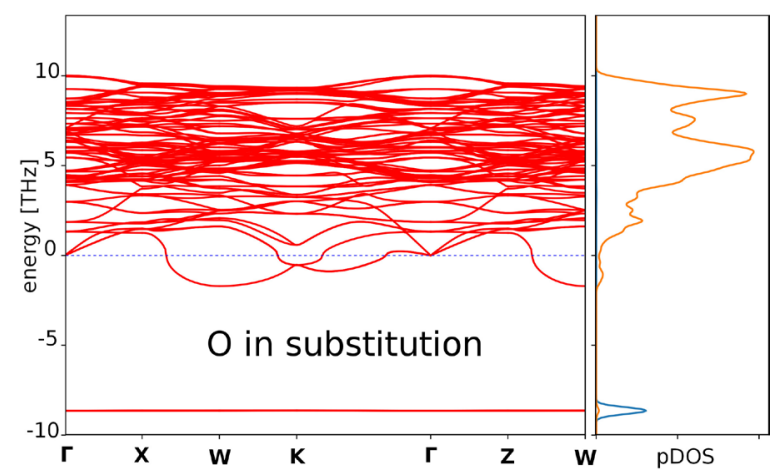

Figure 2. Vibrational band structure (vBAND) and density-ofstates (pDOS) for a system with an $\mathrm{O}$ atom in a substituted position. Negative frequencies mean that the configuration is unstable. The pDOS displays the density-of-states projected on atoms: orange and blue lines correspond to $\mathrm{Al}$ and $\mathrm{O}$ vibrations, respectively. Energies are expressed in THz.

activation energy. The diffusion coefficient formula, which uses a multi-state diffusion formalism based on the work of Landman et al [24] is finally given and results are compared to $\mathrm{kMC}$ simulations and literature.

\section{Computational details}

Atomistic calculations were performed with the DFT using the Vienna ab initio simulation package [25]. We used the Perdew-Burke-Ernzerhof [26] functional and projector augmented-wave pseudo-potentials [27]. We employed $3 \times 3 \times 3$ supercells (i.e. 108 atoms per unit-cell) to study the stability of sites. The inter-atomic forces were fully relaxed and calculations were performed at zero pressure (supercell shapes were fully relaxed). The plane-wave cutoff energy was set to $600 \mathrm{eV}$, and $8 \times 8 \times 8$ MonkhorstPack mesh grids [28] were used to sample the first Brillouin zone. These criteria give formation energies converged to within $2-3 \mathrm{meV}$. Results on reference states are summarized in appendix $\mathrm{A}$. 

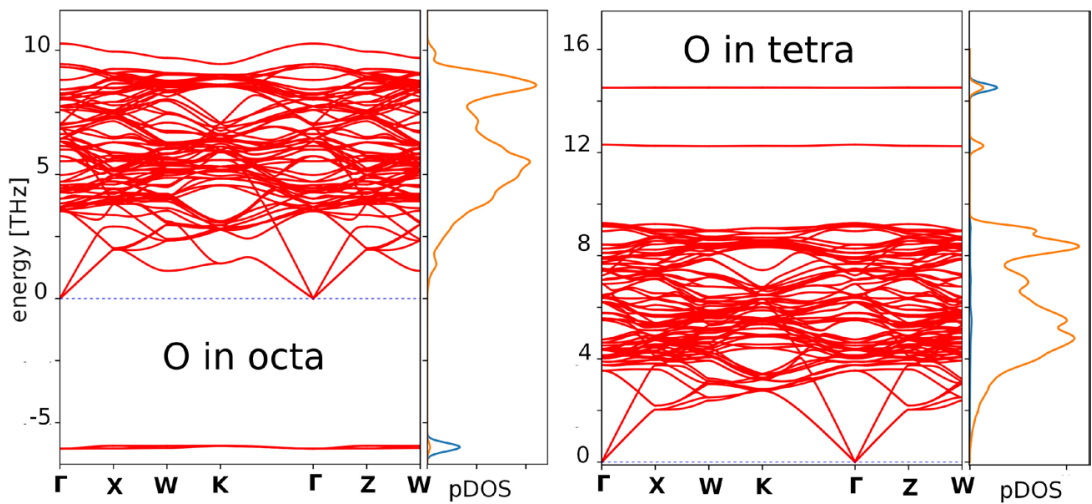

Figure 3. vBAND and pDOS plots for aluminum with one oxygen either in an $o$ site (left) or in a $t$ site (right).
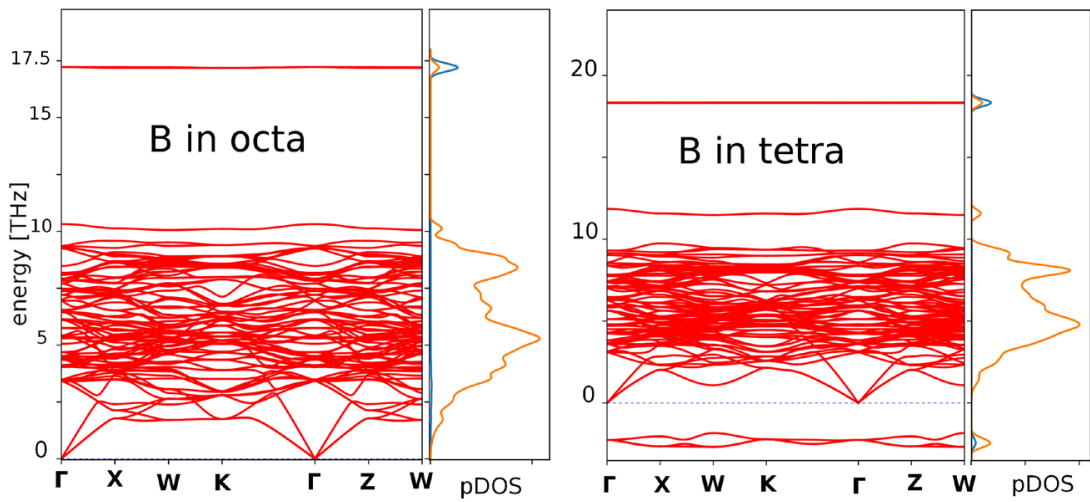

Figure 4. vBAND and pDOS plots for aluminum with one boron either in an $o$ site (left) or in a $t$ site (right).

The inter-atomic force constants of each configuration were obtained as finite differences of forces with respect to atomic displacements (using phonopy package [29]). Due to excessive computational costs, these calculations were only done on $2 \times 2 \times 2$ super-cells. Nevertheless, some tests were conducted on larger supercells and led to similar results quantitatively speaking (see below).

From these vibrational properties, we computed the vibrational free energy $G^{\mathrm{vib}}$, expressed as:

$$
G^{\mathrm{vib}}(T)=k_{\mathrm{B}} T \sum_{n, q} \ln \left[2 \sinh \left(\frac{\hbar \omega_{n, q}}{2 k_{\mathrm{B}} T}\right)\right]
$$

where $\omega_{n, q}$ are the supercell frequencies. In this work, frequencies were only calculated at $0 \mathrm{~K}$ in first-order approximation. The summations were performed on $20 \times 20 \times 20$ q-meshes. The zero-point energy (ZPE) is thus expressed as:

$$
\mathrm{ZPE}=G_{\mathrm{bulk}+\mathrm{X}}^{\mathrm{vib}}(0)-G_{\mathrm{bulk}}^{\mathrm{vib}}(0)-G_{\mathrm{refX}}^{\mathrm{vib}}(0)
$$

where $G_{\text {refX }}^{\mathrm{vib}}$ is the vibrational free energy of $\mathrm{X}$ in its reference state, i.e. molecule (for $\mathrm{H}, \mathrm{N}$ and $\mathrm{O}$ ) or crystal (carbon diamond and hR12-Boron).

To compute diffusion coefficients, the atomistic mechanisms are characterized by constant escape frequencies, from $x$ to $y, \Gamma_{x y}$ :

$$
\Gamma_{x y}(T)=\nu_{x y}(T) \exp \left(-E_{x y}^{m} / k_{\mathrm{B}} T\right) .
$$

$E_{x y}^{m}$ is the migration energy from $x$ to $y$ positions and $\nu_{x y}$ is the attempt frequency of the jump. To compute $E_{x y}^{m}$, CLIMB-NEB calculations [30] were conducted on $2 \times 2 \times 2$ and $3 \times 3 \times 3$ supercells (supercell shapes were not relaxed). The individual rates from $x$ to $y$ positions (where $x$ and $y=t, o$ or $M$ ), $\nu_{x y}(T)$ are given by:

$$
\nu_{x y}(T)=\frac{k_{\mathrm{B}} T}{h} \exp \left[\frac{\Delta G_{x y}^{\mathrm{vib}}(T)}{k_{\mathrm{B}} T}\right]
$$

where $\Delta G_{x y}^{\mathrm{vib}}(T)$ is the vibrational free energy difference between the initial position $(x)$ and the transition state $(t s)$, $\Delta G_{x y}^{\mathrm{vib}}(T)=G_{x}^{\mathrm{vib}}(T)-G_{t s}^{\mathrm{vib}}(T)$ (summation done with real frequencies). It is often approximated by its high temperature expression, $\nu_{x y}^{*}$ (see Wimmer for details [9]):

$$
\nu_{x y}^{*} \simeq \frac{\prod_{n=1}^{3 N-3} \omega_{q=0, n}^{x}}{\prod_{n=1}^{3 N-4} \omega_{q=0, n}^{t s}} .
$$

Hereinafter, we use both expressions: equation (4) in the model and equation (5) in $\mathrm{kMC}$ simulations.

\section{Results}

\subsection{Stability}

The formation energies $\left(H_{f}[X]\right)$ of the $X$ atoms in interstitial positions, expressed by:

$$
H_{f}[X]=E_{o}[M . A l+X]-E_{o}[M \cdot A l]-E_{o}[\operatorname{ref}-\mathrm{X}],
$$

are summarized in table $1 . E_{o}$ are the energies of the system with and without interstitial elements. $E_{o}[\operatorname{ref}-\mathrm{X}]$ is the energy per atom of the reference state of $X$, i.e. the molecule 

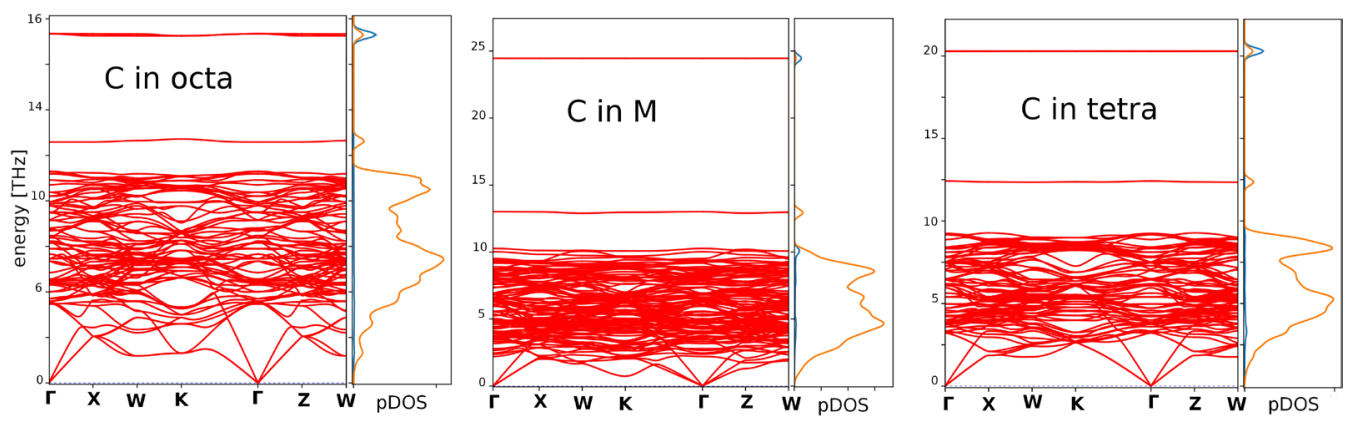

Figure 5. vBAND and pDOS plots for aluminum with one carbon in $o, M$ and $t$ sites (from left to right.

for $\mathrm{H}, \mathrm{O}$ and $\mathrm{N}$ atoms, and the diamond and $\mathrm{hR} 12$ structures (space group No 166) for carbon and boron, respectively. We also give the ZPE, which were computed using the frequencies of the $X$ atom in the metal and in the reference states (equation (2)), the Voronoï volume (in $\AA^{3}$ ) [31] and the volumes of formation $\left(\Omega_{f}\right)$.

We found that the substituted positions (i.e. instead of an $\mathrm{Al}$ atom) are always unstable. The analysis of frequencies shows that there are always three imaginary frequencies associated with the interstitial element as illustrated on figure 2 , in the case of $\mathrm{O}$ atom. In the following, we consider that species prefer to be located in interstitial positions. The formation of clusters with vacancy is out of the scope of this work. Contrary other fcc structures (like nickel for instance), we found that the most stable positions and the intrinsic stability of the sites $(o$ and $t$ ) are strongly dependent on the specie.

The difference in energy between $t$ and $o$ sites $\left(\Delta H=\left\|H_{f}[t]-H_{f}[o]\right\|\right)$ for $\mathrm{H}$ atoms is low, i.e. $\Delta H \simeq 24$ $\mathrm{meV}$ (including the ZPE), while it is higher for $\mathrm{N}$ atoms $(\Delta H \simeq 793 \mathrm{meV})$. The results on $\mathrm{H}$ atoms are in excellent agreement with the literature [7]. Even if $t$ sites are also the stable positions for $\mathrm{O}$ atoms, the case of oxygen must be treated separately. Calculated frequencies show that there is only one stable position: tetrahedral sites. The $o$ position is unstable as illustrated in figure 3. No additional stable configuration was found.

In the case of $\mathrm{C}$ and $\mathrm{B}$ atoms, $o$ sites are found energetically more stable than $t$ sites, contrary to previous species. For B atoms, vBAND analysis reveals that $t$ sites are unstable (see figure 4). Moreover, contrary to other fcc structures, we found one additional stable position for carbon: the position labeled $M$ (for middle), see figure 1 . This configuration corresponds to the half-way position between two first-nearest neighboring ( $\operatorname{lnn}$ ) octahedral positions (o-o). From a structural point of view, $M$ is also the half-way position between two Inn tetrahedral positions $(t-t)$. Although unstable for $\mathrm{H}$, $\mathrm{N}, \mathrm{O}$ atoms (two imaginary frequencies) and $\mathrm{B}$ atoms (one imaginary frequency), this configuration is stable for $\mathrm{C}$ atoms (see figure 5). However, its formation energy is higher than other sites (see table 1).

To try to explain the relative stability of the sites, we first focus on the steric effects by discussing volumes of Voronoï [31] $\left(\mathcal{V}_{\text {oro }}\right)$ and formation $\left(\Omega_{f}\right)$ listed in table 1. Atoms with a large atomic radius (B and $\mathrm{C}$ atoms) are preferably located in $o$ sites, which are the smallest ones, while other atoms are in $t$ sites. This suggests that $\mathrm{C}$ and $\mathrm{B}$ atoms strongly interact with

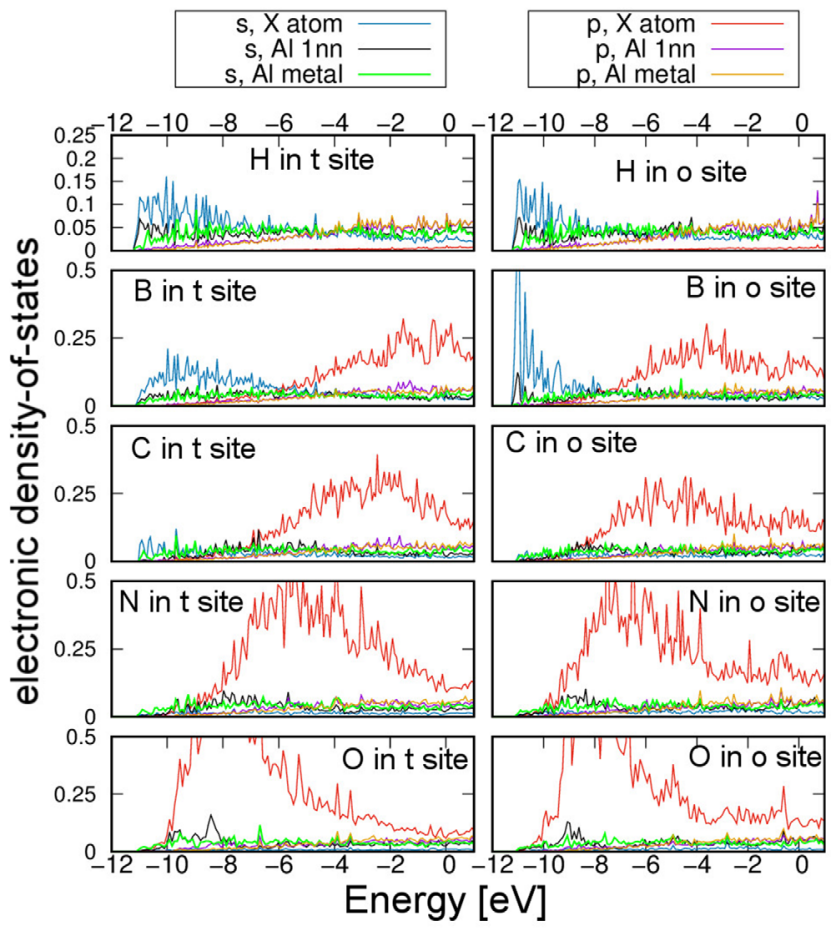

Figure 6. eDOS plot projected onto $s$ and $p$ shells: we displayed the contributions of interstitial elements and of its first-nearest neighboring $\mathrm{Al}$ atoms. We also represented the contribution of one $\mathrm{Al}$ atom located far away from the interstitial.

$\mathrm{Al}$ atoms. $\delta \mathcal{V}_{\text {oro }}$, which corresponds to the site's local distortion when filled, and $\Omega_{f}$ the global effect on the lattice, clearly show the influence of the insertion. In the case of B atoms, both volumes are large, specially in $t$ sites, which would explain why this configuration is unstable. In other cases, we clearly identify the steric effects, but it is difficult to explain why $o$ sites are unstable for $\mathrm{O}$ atoms, while $o, t$ and $M$ sites are stable for $\mathrm{C}$ atoms.

In addition, we plotted the electronic density-of-states (eDOS) in figure 6 (projected onto $\mathrm{Al}$ and $\mathrm{X}$ atoms). $\mathrm{H}$ atoms, which are small atoms, only interacts with $\mathrm{Al}$ atoms with their $s$ states, thus explaining why both sites have equivalent energies. The charge transfer is only located between $\mathrm{Al}$ and $\mathrm{H}$ atoms as we can see in the plot of the differential charge density maps displayed in figure 7 . For $\mathrm{O}, \mathrm{N}$ and $\mathrm{C}$ atoms, where $s$ shells are located deeper in energy $(-21,-14$ and $-13 \mathrm{eV}$, respectively), they only interact with $\mathrm{Al}$ atoms with their $p$ states, contrary to what was found for B atoms. From C to $\mathrm{O}$ atoms, the electronegativity of these guest atoms induces stronger interactions, and therefore stronger bonds. 


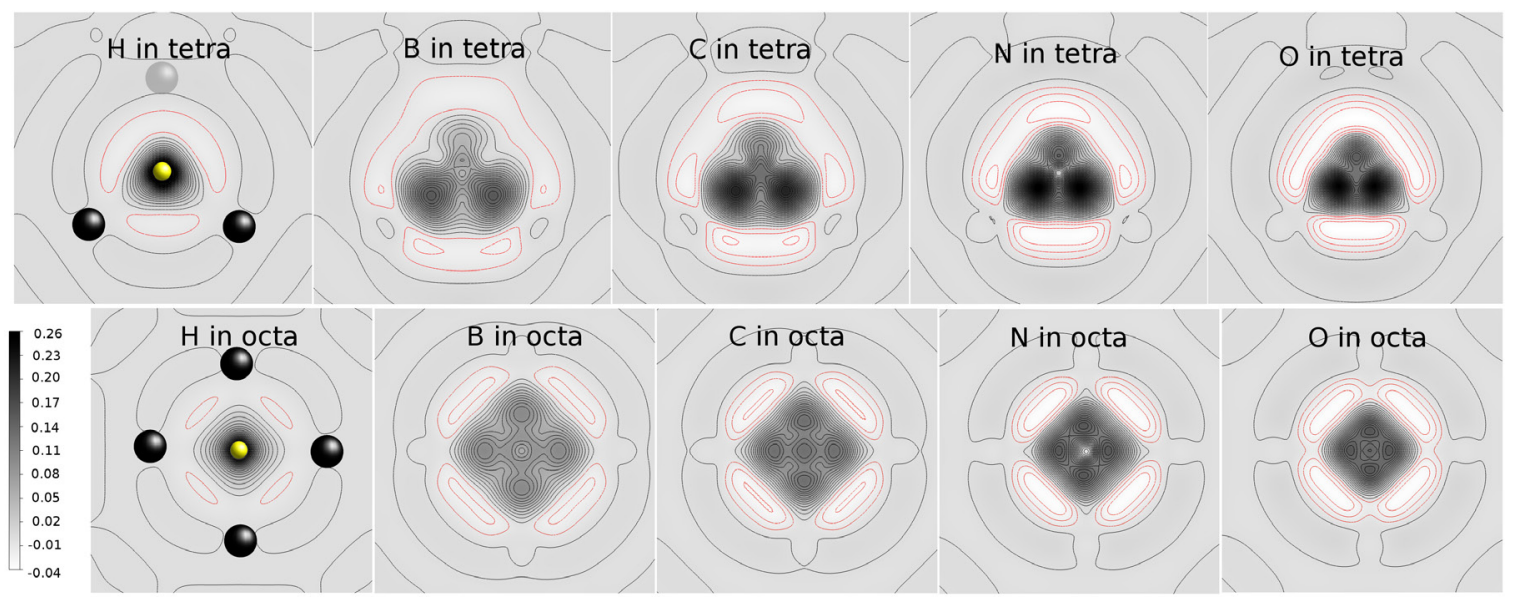

Figure 7. Plot of the contour maps of the charge transfer when sites are filled $(\Delta \rho=\rho[$ bulk $+\mathrm{X}]-\rho[$ bulk $]-\rho[\mathrm{X}])$ : X in tetrahedral sites (top) and in octahedral sites (bottom). Black and yellow balls represent $\mathrm{Al}$ and $\mathrm{X}$ atoms respectively. The same scale is used for all figures.

If we look at the eDOS of $o$ sites in detail, in the case of $\mathrm{N}$ and $\mathrm{O}$ atoms, we note an increasing of the charge density around the Fermi level (located in $0 \mathrm{eV}$ ) in comparison to the eDOS of the $t$ sites. This suggests that, when $\mathrm{O}$ and $\mathrm{N}$ atoms are located in $o$ sites, they should embrittle Al bonds. On the other hand, for $\mathrm{C}$ and $\mathrm{B}$ atoms, where hybridization with aluminium orbitals is at higher energy, the increase in charge density at the Fermi level is observed for $t$ sites. These results can therefore be correlated with the results presented above on site stability.

\subsection{Diffusion mechanisms}

In the light of these results, we hereinafter discuss the diffusion mechanisms of these species. As for stability, diffusion mechanisms are dependent on the specie. In fcc systems, three different paths were generally considered: the first path is between one tetrahedral site and one octahedral site $(t-o)$, the second is between two Inn $t$ positions $(t-t)$, and the third between two Inn $o$ positions $(o-o)$. In the case of carbon, since $M$ positions are stable, additional paths were considered: along $t-M$ and $o-M$ directions. CLIMB-NEB calculations [30] were used to look for the transition states, obtain the energy landscape and compute the migration energies $\left(E^{m}\right)$. Results (attempt frequencies calculated at high temperature, $\nu_{x y}^{*}$, and migration energies) are listed in table 2 . The corresponding energy landscapes are displayed in figure 8 . Vibrational band structures of transition states, conducted on $2 \times 2 \times 2$ supercells, are plotted in appendix B. To test the accuracy of migration energies, we directly computed the transition state energies of of $\mathrm{B}$ and $\mathrm{O}$ atoms. Their atomic position and supercell shapes were fully relaxed. By symmetry, in $M$ and in the transition state of $\mathrm{O}$ atoms, the atoms can not fall in their stable site. We found that the effects of shapes relaxation was low (about $50 \mathrm{meV}$ ): CLIMB-NEB simulation give 1.16 and $0.75 \mathrm{eV}$, for $\mathrm{O}$ and $\mathrm{B}$ atoms respectively, while direct simulations gave 1.10 and $0.75 \mathrm{eV}$.

The case of hydrogen is a well-known one: the only path is between $t$ and $o$ sites. The transition state is almost located
Table 2. Migration energies $E^{m}$ (in $\mathrm{eV}, \mathrm{OK}$ value) and attempt frequency, $\nu_{x y}^{*}$ (in THz), approximated at high temperature of different paths.

\begin{tabular}{llllll}
\hline & & $t-t(o-o)$ & $o-t(t-o)$ & $o-M(M-o)$ & $t-M(M-t)$ \\
\hline $\mathrm{H}$ & $E^{m}$ & - & $0.06(0.17)$ & - & - \\
& $\nu_{x y}^{*}$ & - & $1.0(10.0)$ & - & - \\
$\mathrm{B}$ & $E^{m}$ & $-(0.75)$ & - & - & - \\
& $\nu_{x y}^{*}$ & $-(40.9)$ & - & - & - \\
$\mathrm{C}$ & $E^{m}$ & - & $0.60(0.23)$ & $0.63(0.06)$ & $0.23(0.02)$ \\
& $\nu_{x y}^{*}$ & - & $0.1(0.05)$ & $13.7(1.0)$ & $27.2(4.4)$ \\
$\mathrm{N}$ & $E^{m}$ & - & $0.09(0.92)$ & - & - \\
& $\nu_{x y}^{*}$ & - & $0.72(20.4)$ & - & - \\
$\mathrm{O}$ & $E^{m}$ & 1.16 & - & - & - \\
& $\nu_{x y}^{*}$ & 310.5 & - & - & - \\
\hline
\end{tabular}

in the middle of the path, when the $\mathrm{H}$ atom crosses the triangle formed by $\mathrm{Al}$ atoms. The migration energies we found are in excellent agreement with earlier theoretical literature: Wolverton [7] also found $0.17 \mathrm{eV}$ for the migration energy from $t$ to $o$ (obtained on $2 \times 2 \times 2$ supercells).

We obtain similar result for $\mathrm{N}$ atoms, but their migration energy (along $t \Rightarrow o$ ) is significantly higher than the one of $\mathrm{H}$ atoms $(0.92 \mathrm{eV})$. Moreover, the position of the transition state is found slightly out of the direct path (offset of the ideal position).

For $\mathrm{O}$ atoms, since the $o$ sites are unstable, only a direct $t-t$ path is possible. We found that the transition state is not located in $M$ (the half-way position) but in the barycenter of the triangle (white ball, figure 1) composed of two $\mathrm{Al}$ atoms and one octahedral site. There are therefore two equivalent paths for one jump between Inn $t$ sites (one for each triangle located left and right of the diagonal bounds between the two first-nearest neighboring $\mathrm{Al}$ atoms). $\mathrm{O}$ atoms have thus twelve $(6 \times 2)$ paths to move from a $t$ site to another $t$ site (the length of the jump is thus slightly increased).

The case of $\mathrm{B}$ atoms is similar to the $\mathrm{O}$ atoms, however, $\mathrm{B}$ atoms only move between $o$ sites, and the transition state is located in $M$. 


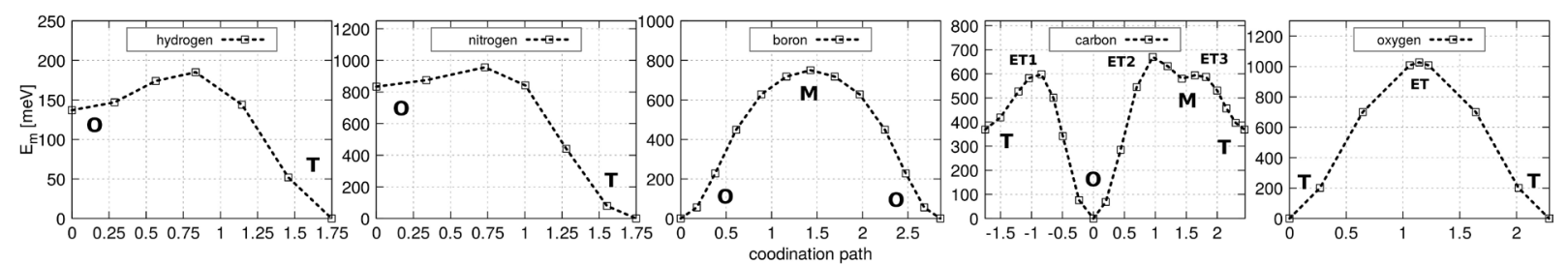

Figure 8. Plot of the energy landscape obtained using NEB calculations in the case of $\mathrm{H}, \mathrm{N}, \mathrm{B}, \mathrm{C}$ and $\mathrm{O}$ atoms (left to right). In the case of carbon, there are three transition states labeled $E T_{1}, E T_{2}$ and $E T_{3}$.
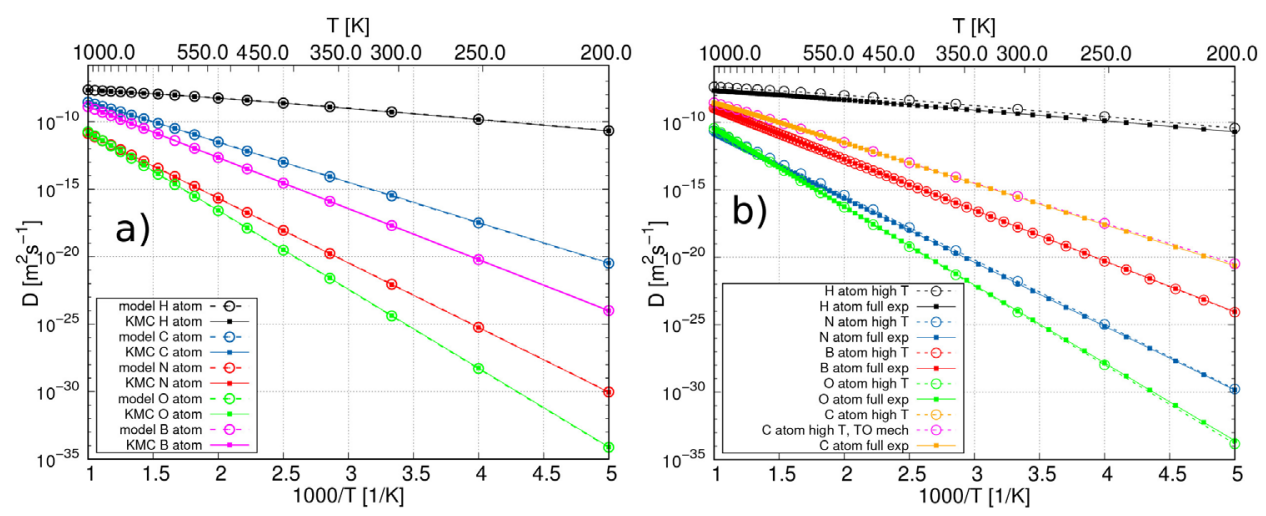

Figure 9. Plot of the diffusion coefficient of H, C, N, O and B atoms, comparison with kMC simulations and model (figure (a)). Figure (b) represents the diffusion coefficient using the model where the attempt frequency is either approximated by its high temperature expression, $\nu_{x y}^{*}$ (circles), or performed with the full expression $\nu_{x y}(T)$ (small squares), see text. For information, the melting point of fcc-Al is about $960 \mathrm{~K}$.

Finally, for $\mathrm{C}$ atoms, the energy landscape is different from previous species. NEB simulations give us three transition states: along $t-o, t-M$ and $o-M$. In the first case, the path is found to be direct. Along $t-M$ and $o-M$ the jumps are located near the $M$ position and the transition state is slightly out the direct path. We could also note that the attempt frequency of $\mathrm{C}$ atoms along $t$-o $(0.05$ and 0.1$)$ is lower than those obtained along the other directions. This is due to the additional soft modes in the band structure (see vibrational band structures in appendix B).

\subsection{Diffusion equations}

Using these DFT values, we then computed diffusion coefficients using $\mathrm{kMC}$ simulations (see details in appendix C). We used the same method as described by Wang et al [11].

Results of simulations are depicted in figure 9. To approve our kMC simulations, we extracted explicit expressions of the diffusion mechanisms in each case, according to the method proposed by Landman et al [24] as shown in appendix D. Diffusion equations are thus given by:

$$
\begin{gathered}
D_{o o}=a_{o}^{2} \Gamma_{o o} \\
D_{t t}=\frac{\zeta}{4} a_{o}^{2} \Gamma_{t t} \\
D_{t o}=\frac{1}{2} a_{o}^{2} \frac{\Gamma_{t o} \Gamma_{o t}}{\Gamma_{t o}+2 \Gamma_{o t}} .
\end{gathered}
$$

Table 3. Activation energies (in $\mathrm{eV})$ and the prefactor $D_{o}\left(10^{-6} \mathrm{~m}^{2}\right.$ $\mathrm{s}^{-1}$ or $10^{-10} \mathrm{~cm}^{2} \mathrm{~s}^{-1}$ ) obtained using a fit on a range of $[200 ; 1000]$ $\mathrm{K}$ of the full diffusion coefficient model. According to how the fit was done (direct or logarithmic fit), accuracy of $E_{a}$ is $\pm 10 \mathrm{meV}$. In the case of oxygen, one must take into account the double degeneracy of the path.

\begin{tabular}{llllll}
\hline & $\mathrm{H}$ & $\mathrm{N}$ & $\mathrm{O}$ & $\mathrm{B}$ & $\mathrm{C}$ \\
\hline$E_{a}$ & 0.14 & 0.91 & 1.16 & 0.75 & 0.57 \\
$D_{o}$ & 0.10 & 0.57 & $24.6 \times 2$ & 6.64 & 1.89 \\
\hline
\end{tabular}

When the $t$ and $M$ sites are sufficiently deep, the diffusion atom temporarily equilibrates in both sites, $D_{t o m}$ is thus expressed by:

$$
\begin{aligned}
D_{t o m}= & \frac{a_{o}^{2}}{4}\left[6 \Gamma_{m o} \Gamma_{o m} \Gamma_{t m}+3 \Gamma_{m t} \Gamma_{o m} \Gamma_{t m}+6 \Gamma_{m o} \Gamma_{o t} \Gamma_{t m}\right. \\
& +2 \Gamma_{m t} \Gamma_{o t} \Gamma_{t m}+4 \Gamma_{m o} \Gamma_{o m} \Gamma_{t o}+6 \Gamma_{m t} \Gamma_{o m} \Gamma_{t o} \\
& \left.+4 \Gamma_{m o} \Gamma_{o t} \Gamma_{t o}+4 \Gamma_{m t} \Gamma_{o t} \Gamma_{t o}\right] /\left[6 \Gamma_{m t} \Gamma_{o m}\right. \\
& +4 \Gamma_{m o} \Gamma_{o t}+4 \Gamma_{m t} \Gamma_{o t}+3 \Gamma_{m o} \Gamma_{t m}+18 \Gamma_{o m} \Gamma_{t m} \\
+ & \left.12 \Gamma_{o t} \Gamma_{t m}+2 \Gamma_{m o} \Gamma_{t o}+2 \Gamma_{m t} \Gamma_{t o}+12 \Gamma_{o m} \Gamma_{t o}\right] .
\end{aligned}
$$

$a_{o}$ is the lattice parameter of the fcc system, set to $4.04 \AA$, and $\Gamma_{x y}$ given by equations (3) and (4).

Equations $(7 a)$ and $(7 b)$ correspond to the cases where the initial and final positions are the $o$ and the $t$ sites, respectively. For $\mathrm{O}$ (along $t-t$ ), as there are two paths for each trajectory, $\zeta$ is equal to 2. When $t$ and $o$ are stable positions (for $\mathrm{H}$ and $\mathrm{N}$ atoms) and there is only one path, equation (7c) 
must be applied (equivalent expression to the one proposed by Wimmer et al [9]). Finally, in the case of carbon, where there are three paths and three stable positions, the coefficient of diffusion follows the equation (D.6). The full expression, includes all jumps.

As we can see in figure 9(a), our kMC simulations and these formulas lead to the same results even for $\mathrm{C}$ atoms. Except for hydrogen, the diffusion of interstitial species is slow in aluminum, $D$ is lower than $10^{-10} \mathrm{~m}^{2} \mathrm{~s}^{-1}$, especially for $\mathrm{O}$ and $\mathrm{N}$ atoms. The lack of experimental values does not enable us to validate our results, especially in the case of $\mathrm{C}$ atoms, for which the accuracy of the attempt frequency along $t$ - $o$ could be improved. Nonetheless, these explicit formulas will now be used to easily describe atomistic mechanisms. In the case of $\mathrm{C}$ atoms, if we consider only $t-o$ paths, we reproduce the diffusivity matches (see magenta circles in figure 9(b)). Finally, the $M$ configuration does influence the diffusion mechanism of $\mathrm{C}$ atoms (not stable enough).

The effect of temperature on the attempt frequency $\left(\nu_{x y}^{*}\right.$ and $\nu_{x y}(T)$ ) was investigated. It was found relatively low, see figure 9(b), even in the case of the $\mathrm{H}$ atoms. From these results, we fitted the diffusivities by means of an Arrhenius law $\left(D=D_{o} \exp \left(-E_{a} / k_{\mathrm{B}} T\right)\right)$ to obtain $D_{o}$ and $E_{a}$ values. The Arrhenius law allows reproducing with a high accuracy exact diffusivity curves, showing that, in the case of aluminum, the temperature dependence on activation energies is low (neglecting the thermal expansions). Results are summarized in table 3.

As explained previously, only $\mathrm{H}$ diffusivity was studied. The activation energy value that was found $(0.14 \mathrm{eV}$, fitted on the diffusivity) is slightly lower than the experimental one $(0.17 \mathrm{eV},[18]$ and references therein). The activation energy we found includes both migration energies $(t-o$ and $o-t)$, that which explains why our value is slightly different from the migration energies. Wolverton's study leads to the same result as ours. Our value of $E_{a}$ is however smaller than the experimental findings. This discrepancy can be explained by a slow down in the diffusivity induced by the formation of $\mathrm{VH}$ cluster even at low $\mathrm{H}$ concentration, as explained by Tanguy [32].

\section{Conclusion}

In conclusion, we showed that while $\mathrm{H}$ and $\mathrm{N}$ atoms exhibit a 'simple' diffusion mechanism (along a direct $t$ - $o$ path), the cases of $\mathrm{O}, \mathrm{C}$ and $\mathrm{B}$ atoms demand a special focus. For $\mathrm{C}$ atoms a new stable position in the network has been identified and the mechanism of diffusion therefore differs from what was expected. A complex path occurs for these elements. The case of $\mathrm{O}(\mathrm{B})$ atoms involves a direct $t-t(o-o)$ diffusion, with a saddle point different from what is generally expected.
Table A1. Cohesion energies ( $E_{\mathrm{coh}}$, in eV/atom) and lattice parameters $\left(a_{o}\right.$, in $\AA$ ), or inter-atomic distances $(d$, in $\AA$ ) of reference states.

\begin{tabular}{|c|c|c|c|c|c|c|}
\hline & & $\mathrm{H}_{2}$ & $\mathrm{O}_{2}$ & $\mathrm{~N}_{2}$ & C-dia & B- $\alpha$ \\
\hline$E_{\mathrm{coh}}$ & & 2.27 & 3.25 & 5.19 & 7.85 & 6.37 \\
\hline Exp. & {$[34,35]$} & 2.21 & 2.59 & 4.88 & 7.37 & 5.81 \\
\hline $\mathrm{d} / a_{o}$ & & 0.75 & 1.23 & 1.11 & 3.55 & $2.83 / 4.18$ \\
\hline Exp. & [35] & 0.74 & 1.21 & 1.10 & 3.57 & - \\
\hline
\end{tabular}

Finally, explicit diffusion coefficients formulas, confirmed by kMC simulations, have been presented and discussed. A new focus should now be put onto other fcc structures, especially for atoms bonding with metals.

\section{Acknowledgments}

This work was performed using HPC resources from CALMIP (Grant 2017-p0842) and GENCI-CINES (Grant 2016-c2016097722). Authors acknowledge D Tanguy and A Berthomieu for their remarks and their advice.

\section{Appendix A. Molecular references}

We report here cohesion energies ( $E_{\mathrm{coh}}$, in $\mathrm{eV}$ per atom) of reference states: molecules for $\mathrm{H}, \mathrm{N}$ and $\mathrm{O}$ atoms and crystals for $\mathrm{C}$ (diamond) and B atoms. For boron, we used the hR12 structure $(\alpha)$, space group No 166 [33]. Our results, listed in table A1, are consistent with experimental and theoretical values (see for example [34] and references therein) to the extent that the DFT is inefficient to describe the ground state of atoms. Atomic distance and lattice parameters correspond well with experimental ones.

\section{Appendix B. Vibrational properties}

Vibrational properties were computed on $2 \times 2 \times 2$ supercells using a finite displacement method (phonopy [29]). $8 \times 8 \times 8 \mathbf{k}$-meshes grids and $600 \mathrm{eV}$ as cut-off energy were used to compute forces. In the case of transition states, one obtains only one imaginary frequency (here one negative frequency). Figure B1 represent the vibrational band structures of the transition states in the case of $\mathrm{H}, \mathrm{B}, \mathrm{N}$ and $\mathrm{O}$ atoms. Figure B2 are those of the $\mathrm{C}$ atoms.

\section{Appendix C. Detail of kMC simulations}

Kinetic Monte Carlo simulations were carried out to estimate the coefficient of diffusion of interstitial elements and validate 

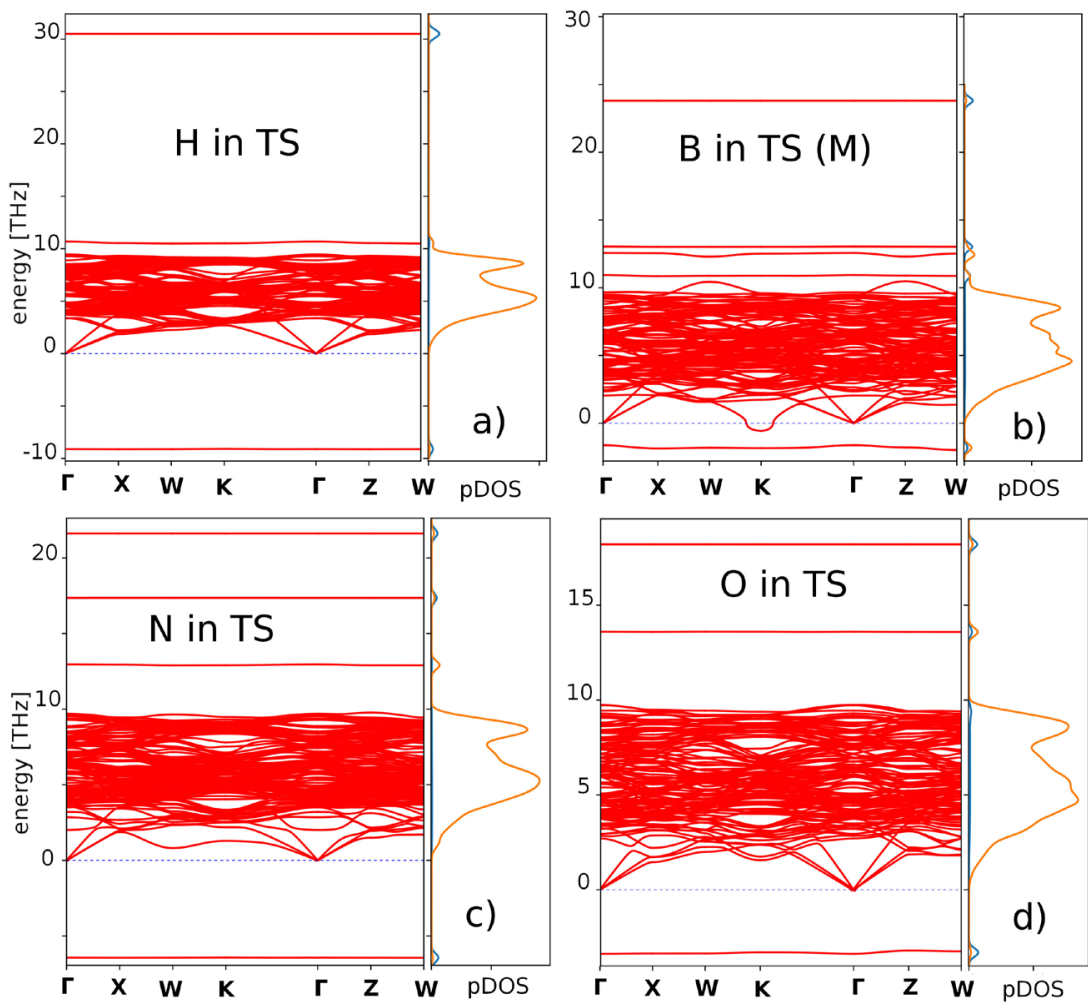

Figure B1. vBAND and pDOS plots for transition states of transition states of $\mathrm{H}, \mathrm{B}, \mathrm{N}$ and $\mathrm{O}$ atoms.
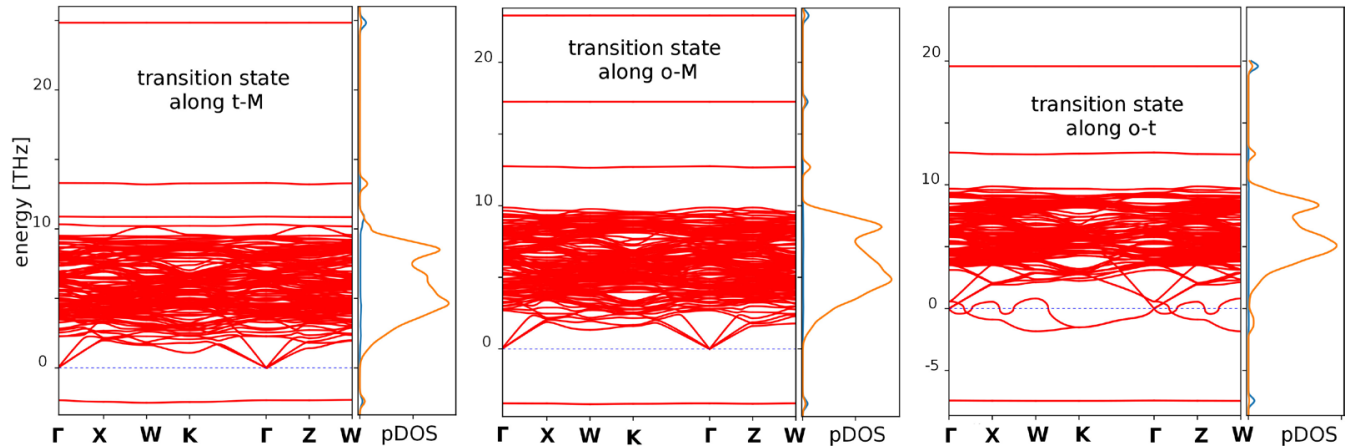

Figure B2. vBAND and $\mathrm{pDOS}$ plots for transition states of $\mathrm{C}$ atoms.

the models. The diffusions were considered as Poisson processes. The constant escape frequency $\Gamma$, is the sum of the rates of all possible jumps. In first-order approximation, we used $\nu_{x y}^{*}$ instead of $\nu_{x y}(T)$ (in the model and $\mathrm{kMC}$ ). $500 \mathrm{kMC}$ simulations with 500 millions of steps per $\mathrm{kMC}$ were used.

\section{Appendix D. Multistate model}

\section{D.1. $t$-o diffusion}

In the case where there are $t$ and $o$ sites and only one jump (along the $t-o$ ), $D_{t o}$ is expressed by:

$$
D_{t o}=\frac{1}{2} a_{o}^{2} \frac{\Gamma_{t o} \Gamma_{o t}}{\Gamma_{t o}+2 \Gamma_{o t}} .
$$

To derive this equation, we used the multi-state diffusion formalism proposed by Landman et al [24]. We must first identify the number of non-equivalent position in the primitive cell: tetrahedral sites are in $8 c$ positions and octahedral sites in $4 b$. In the primitive cell there are thus two inequivalent $t$ sites (labeled $t_{1}$ and $t_{2}$ ) and one $o$ site. We built two quantities: the Laplace transform of the waiting time density matrix $(\psi(u))$ and the Fourrier transform matrix of the displacements of $X$ in $\mathrm{Ni}(p(k)) . \psi(u)$ is given by:

$$
\underline{\psi}(u)=\begin{aligned}
& o_{1} \\
& o_{1} \\
& t_{1} \\
& t_{2}
\end{aligned} \quad\left[\begin{array}{ccc}
t_{1} & t_{2} \\
0 & \frac{4 \Gamma_{t o}}{4 \Gamma_{t o}+u} & \frac{4 \Gamma_{t o}}{4 \Gamma_{t o}+u} \\
\frac{4 \Gamma_{o t}}{8 \Gamma_{o t}+u} & 0 & 0 \\
\frac{4 \Gamma_{o t}}{8 \Gamma_{o t}+u} & 0 & 0
\end{array}\right]
$$

where $\Gamma_{x y}$ are the rate of escape from internal state $x$ to state $y$. Here the jumps are: $o \Longrightarrow t_{1}, o \Longrightarrow t_{2}, t_{1} \Longrightarrow o$ and $t_{2} \Longrightarrow o$. $\underline{p}(k)$ is given by: 


$$
\underline{p}(k)=\begin{aligned}
& o_{1} \\
& o_{1} \\
& t_{1} \\
& t_{2}
\end{aligned} \quad\left[\begin{array}{ccc}
0 & t_{1} & t_{2} \\
A_{t_{1} o} & A_{t_{2} o} \\
A_{o t_{2}} & 0 & 0
\end{array}\right]
$$

where

$$
\begin{aligned}
& A_{t_{1} o}=\left[1+\mathrm{e}^{-\mathrm{i} l k_{1}}+\mathrm{e}^{-\mathrm{i} l k_{2}}+\mathrm{e}^{-\mathrm{i} l k_{3}}\right] / 4 \\
& A_{o t_{1}}=\left[1+\mathrm{e}^{\mathrm{i} l k_{1}}+\mathrm{e}^{\mathrm{i} l k_{2}}+\mathrm{e}^{\mathrm{i} l k_{3}}\right] / 4 \\
& A_{t_{2} o}=\left[1+\mathrm{e}^{\mathrm{i} l k_{1}}+\mathrm{e}^{\mathrm{i} l k_{2}}+\mathrm{e}^{\mathrm{i} l k_{3}}\right] / 4 \\
& A_{o t_{2}}=\left[1+\mathrm{e}^{-\mathrm{i} l k_{1}}+\mathrm{e}^{-\mathrm{i} l k_{2}}+\mathrm{e}^{-\mathrm{i} l k_{3}}\right] / 4
\end{aligned}
$$

where $l=a_{o} \sqrt{2} / 2$.

We then followed the same approach than Wu et al [36] to derive equations.

\section{D.2. Three sites diffusion}

In the case where there are three sites $(t, o$ and $M)$, three paths were considered: $t-o, t-M$ and $o-M$. In addition to the previous case, there are six additional non-equivalent $M$ positions per primitive cell. $p(k)$ matrix is thus equal to:

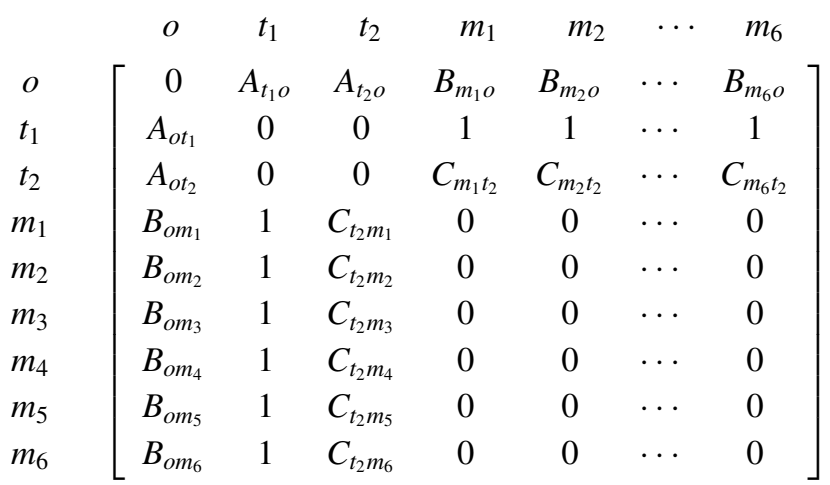

\begin{tabular}{|c|c|c|c|c|c|c|c|}
\hline & $O$ & $t_{1}$ & $t_{2}$ & $m_{1}$ & $m_{2}$ & $\cdots$ & $m_{6}$ \\
\hline & 0 & $4 \Gamma_{t o}$ & $\frac{4 \Gamma_{t o}}{\Lambda+u}$ & $\frac{2 \Gamma_{m o}}{}$ & $2 \Gamma_{m o}$ & & $\frac{2 \Gamma_{m o}}{1}$ \\
\hline$O$ & $4 \Gamma_{o t}$ & $\begin{array}{c}n_{t}+u \\
0\end{array}$ & ${ }_{0}^{\Lambda_{t}+u}$ & $\begin{array}{c}\Lambda_{m}+u \\
\Gamma_{m t} \\
\end{array}$ & $\begin{array}{c}\Lambda_{m}+u \\
\Gamma_{m t}\end{array}$ & & $\begin{array}{c}\Lambda_{m}+u \\
\Gamma_{m t}\end{array}$ \\
\hline$t_{1}$ & $\overline{\Lambda_{o}+u}$ & 0 & 0 & $\overline{\Lambda_{m}+u}$ & $\overline{\Lambda_{m}+u}$ & & $\overline{\Lambda_{m}+u}$ \\
\hline$t_{2}$ & $\frac{4 \Gamma_{o t}}{\Lambda_{o}+u}$ & 0 & 0 & $\frac{\Gamma_{m t}}{\Lambda_{m}+u}$ & $\frac{\Gamma_{m t}}{\Lambda_{m}+u}$ & & $\frac{\Gamma_{m t}}{\Lambda_{m}+u}$ \\
\hline$m_{1}$ & $\frac{2 \Gamma_{o m}}{\Lambda_{o}+u}$ & $\frac{\Gamma_{t m}}{\Lambda_{t}+u}$ & $\frac{\Gamma_{t m}}{\Lambda_{t}+u}$ & 0 & 0 & . & 0 \\
\hline$m_{2}$ & $\frac{2 \Gamma_{o m}}{\Lambda+u}$ & $\frac{\Gamma_{t m}}{\Lambda++t}$ & $\frac{\Gamma_{t m}}{\Lambda+u}$ & 0 & 0 & $\ldots$ & 0 \\
\hline$m_{3}$ & $\frac{2 \Gamma_{o m}}{\Lambda_{o}+u}$ & $\frac{\Gamma_{t m}}{\Lambda_{t}+u}$ & $\frac{\Gamma_{t m}}{\Lambda}$ & 0 & 0 & $\cdots$ & 0 \\
\hline$m_{4}$ & $2 \Gamma_{o m}$ & $\Gamma_{t m}$ & $\frac{\Gamma_{t m}}{\Lambda}$ & 0 & 0 & $\cdots$ & 0 \\
\hline$m_{5}$ & $2 \Gamma_{o m}$ & $\Gamma_{t m}$ & $\Gamma_{t m}$ & 0 & 0 & $\ldots$ & 0 \\
\hline$m_{6}$ & $\begin{array}{r}\Lambda_{o}+u \\
2 \Gamma_{o m} \\
\end{array}$ & $\begin{array}{l}t_{t}+u \\
\Gamma_{t m} \\
\end{array}$ & $\begin{array}{l}\Lambda_{t}+u \\
\Gamma_{t m} \\
\end{array}$ & 0 & 0 & $\ldots$ & 0 \\
\hline & $\overline{\Lambda_{o}+u}$ & $\overline{\Lambda_{t}+u}$ & $\Lambda_{t}+u$ & & & & \\
\hline
\end{tabular}

where

$$
\begin{array}{ll}
B_{m_{1} o}=\left[\mathrm{e}^{-\mathrm{i} l k_{2}}+\mathrm{e}^{-\mathrm{i} l k_{3}}\right] / 2 & B_{o m_{1}}=\left[\mathrm{e}^{\mathrm{i} l k_{2}}+\mathrm{e}^{\mathrm{i} l k_{3}}\right] / 2 \\
B_{m_{2} o}=\left[\mathrm{e}^{-\mathrm{i} l k_{1}}+\mathrm{e}^{-\mathrm{i} l k_{3}}\right] / 2 & B_{o m_{2}}=\left[\mathrm{e}^{\mathrm{i} l k_{1}}+\mathrm{e}^{\mathrm{i} l k_{3}}\right] / 2 \\
B_{m_{3} o}=\left[\mathrm{e}^{-\mathrm{i} l k_{1}}+\mathrm{e}^{-\mathrm{i} l k_{2}}\right] / 2 & B_{o_{3}}=\left[\mathrm{e}^{\mathrm{i} l k_{1}}+\mathrm{e}^{\mathrm{i} l k_{2}}\right] / 2 \\
B_{m_{4} o}=\left[1+\mathrm{e}^{-\mathrm{i} l k_{1}}\right] / 2 & B_{o_{m_{4}}}=\left[1+\mathrm{e}^{\mathrm{i} l k_{1}}\right] / 2 \\
B_{m_{5} o}=\left[1+\mathrm{e}^{-\mathrm{i} l k_{2}}\right] / 2 & B_{o m_{5}}=\left[1+\mathrm{e}^{\mathrm{i} l k_{2}}\right] / 2 \\
B_{m_{6} o}=\left[1+\mathrm{e}^{-\mathrm{i} l k_{3}}\right] / 2 & B_{o m_{6}}=\left[1+\mathrm{e}^{\mathrm{i} l k_{3}}\right] / 2 \\
C_{m_{1} t_{2}}=\mathrm{e}^{-\mathrm{i} l\left(k_{2}+k_{3}\right)} & C_{t_{2} m_{1}}=\mathrm{e}^{\mathrm{i} l\left(k_{2}+k_{3}\right)} \\
C_{m_{2} t_{2}}=\mathrm{e}^{-\mathrm{i} l\left(k_{1}+k_{3}\right)} & C_{t_{2} m_{2}}=\mathrm{e}^{\mathrm{i} l\left(k_{1}+k_{3}\right)} \\
C_{m_{3} t_{2}}=\mathrm{e}^{-\mathrm{i} l\left(k_{1}+k_{2}\right)} & C_{t_{2} m_{3}}=\mathrm{e}^{\mathrm{i} l\left(k_{1}+k_{2}\right)} \\
C_{m_{4} t_{2}}=\mathrm{e}^{-\mathrm{i} l k_{1}} & C_{t_{2} m_{4}}=\mathrm{e}^{\mathrm{i} l k_{1}} \\
C_{m_{5} t_{2}}=\mathrm{e}^{-\mathrm{i} l k_{2}} & C_{t_{2} m_{5}}=\mathrm{e}^{\mathrm{i} l k_{3}} \\
C_{m_{6} t_{2}}=\mathrm{e}^{-\mathrm{i} l k_{3}} & C_{t_{2} m_{6}}=\mathrm{e}^{\mathrm{i} l k_{3}}
\end{array}
$$

and the waiting time density matrix, $\psi(u)$ : where $\quad \Lambda_{o}=12 \Gamma_{o m}+8 \Gamma_{o t}, \quad \Lambda_{t}=4 \Gamma_{t o}+6 \Gamma_{t m} \quad$ and $\Lambda_{m}=2 \Gamma_{m o}+2 \Gamma_{m t}$. One thus finds for $D_{t o m}$ :

$$
\begin{aligned}
D_{t o m}= & \frac{a_{o}^{2}}{4}\left[6 \Gamma_{m o} \Gamma_{o m} \Gamma_{t m}+3 \Gamma_{m t} \Gamma_{o m} \Gamma_{t m}+6 \Gamma_{m o} \Gamma_{o t} \Gamma_{t m}\right. \\
& +2 \Gamma_{m t} \Gamma_{o t} \Gamma_{t m}+4 \Gamma_{m o} \Gamma_{o m} \Gamma_{t o}+6 \Gamma_{m t} \Gamma_{o m} \Gamma_{t o} \\
& \left.+4 \Gamma_{m o} \Gamma_{o t} \Gamma_{t o}+4 \Gamma_{m t} \Gamma_{o t} \Gamma_{t o}\right] /\left[6 \Gamma_{m t} \Gamma_{o m}\right. \\
+ & 4 \Gamma_{m o} \Gamma_{o t}+4 \Gamma_{m t} \Gamma_{o t}+3 \Gamma_{m o} \Gamma_{t m}+18 \Gamma_{o m} \Gamma_{t m} \\
+ & \left.12 \Gamma_{o t} \Gamma_{t m}+2 \Gamma_{m o} \Gamma_{t o}+2 \Gamma_{m t} \Gamma_{t o}+12 \Gamma_{o m} \Gamma_{t o}\right]
\end{aligned}
$$

\section{ORCID iDs}

Damien Connétable @ https://orcid.org/0000-0003-3642-780X

\section{References}

[1] Garnier T, Nastar M, Bellon P and Trinkle D R 2013 Solute drag by vacancies in body-centered cubic alloys Phys. Rev. B 88134201

[2] Garnier T, Trinkle D R, Nastar M and Bellon P 2014 Quantitative modeling of solute drag by vacancies in facecentered-cubic alloys Phys. Rev. B 89144202

[3] Magnin T 1997 2nd Int. Conf. on Corrosion Deformation Interactions (Nice: The Institute of Materials)

[4] Young D J, Zhang J, Geers C and Sch M 2011 Mater. Corros. 627

[5] Grabke H 1998 Thermodynamics, mechanisms and kinetics of metal dusting Mater. Corros. 49 303-8

[6] Prillieux A, Jullian D, Zhang J, Monceau D and Young D J 2017 Internal oxidation in dry and wet conditions for oxygen permeability of $\mathrm{Fe}-\mathrm{Ni}$ alloys at 1150 and $1100{ }^{\circ} \mathrm{C}$ Oxid. Met. $87273-83$

[7] Wolverton C, Ozolins V and Asta M 2004 Hydrogen in aluminum: first-principles calculations of structure and thermodynamics Phys. Rev. B 69144109

[8] Jiang C, Wolverton C, Sofo J, Chen L and Liu Z 2004 Phys. Rev. B 69214202

[9] Wimmer E, Wolf W, Sticht J, Saxe P, Geller C, Najafabadi R and Young G 2008 Temperature dependent diffusion 
coefficients from ab initio computations: H, D and T in nickel Phys. Rev. B 77134305

[10] Heinola $\mathrm{K}$ and Ahlgren T 2010 Diffusion of hydrogen in bcc Tungsten studied with first principle calculations J. Appl. Phys. 107113531

[11] Wang Y, Connétable D and Tanguy D 2016 Influence of trap connectivity on $\mathrm{H}$ diffusion: Vacancy trapping Acta Mater. $103334-40$

[12] Wang Y, Connétable D and Tanguy D 2015 Hydrogen influence on diffusion in nickel from first-principles calculations Phys. Rev. B 91094106

[13] Wang Y 2014 Ab initio calculations of $\mathrm{H}$ interactions with defects in fcc metals: crack tip dislocations and vacancies $P h D$ Thesis Institut National Polytechnique de Toulouse

[14] Connétable D, Andrieu E and Monceau D 2015 Firstprinciples nickel database: energetics of impurities and defects Comput. Mater. Sci. 101 77-87

[15] Vekilova O Y, Bazhanov D I, Simak S I and Abrikosov I A 2009 First-principles study of vacancy-hydrogen interaction in Pd Phys. Rev. B 80024101

[16] Amin-Ahmadi B, Connétable D, Fivel M, Tanguy D, Delmelle R, Turner S, Pardoen T, Proost J, Schryvers D and Idrissi H 2016 Dislocation hydrogen interaction mechanisms in hydrided nanocrystalline palladium films Acta Mater. 111 253-61

[17] Korzhavyi P and Sandström R 2014 Monovacancy in copper: rapping efficiency for hydrogen and oxygen impurities Comput. Mater. Sci. 84 122-8

[18] Ai J-H and Scully J R 2013 Hydrogen diffusivity during corrosion of high-purity aluminum Corrosion $69752-67$

[19] Bose S and Grabke H 1978 Diffusion coefficient of carbon in $\mathrm{Fe}-\mathrm{Ni}$ austenite in the temperature range $950-1100 \mathrm{c}$ Z. Met.kd. 698

[20] Grabke H J and Petersen E M 1978 Diffusivity of nitrogen in iron-nickel alloys Scr. Metall. 12 1111-4

[21] Park J-W and Altstetter C 1987 The diffusion and solubility of oxygen in solid nickel Metall. Trans. A 18A 43

[22] Takada J, Yamamoto S, Kikuchi S and Adachi M 1986 Determination of diffusion coefficient of oxygen in $\gamma$-iron from measurements of internal oxidation in $\mathrm{Fe}-\mathrm{Al}$ alloys Metall. Trans. A 17 221-9

[23] Dai J and Song Y 2013 Influence of H, C, N and O impurities on the stability of $\mathrm{Mg}$ and $\mathrm{Al}$ from first-principles calculations Modelling Simul. Mater. Sci. Eng. 21055014

[24] Landman U and Shlesinger M F 1979 Stochastic theory of multistate diffusion in perfect and defective systems. I. Mathematical formalism Phys. Rev. B 19 6207-19

[25] Kresse G and Hafner J 1993 Ab initio molecular dynamics for liquid metals Phys. Rev. B 47 558R

[26] Perdew J, Burke K and Ernzerhof M 1997 Generalized gradient approximation made simple Phys. Rev. Lett. 781396

[27] Kresse G and Joubert D 1999 From ultrasoft pseudopotentials to the projector augmented-wave method Phys. Rev. B 591758

[28] Monkhorst H and Pack J 1976 Special points for the brilluoin zone integrations Phys. Rev. B 135188

[29] Togo A, Oba F and Tanaka I 2008 First-principles calculations of the ferroelastic transition between rutile-type and $\mathrm{Ca}$ Phys. Rev. B 78134106

[30] Henkelman G, Uberuaga B and Jónsson H 2000 A climbing image nudged elastic band method for finding saddle points and minimum energy paths J. Chem. Phys. 1139901

[31] Rycroft C H 2009 Voro + +: a three-dimensional Voronoi cell library in $\mathrm{c}++$ Chaos $\mathbf{1 9} 041111$

[32] Tanguy D $2016 \mathrm{H}$ effects in $\mathrm{Al}-\mathrm{Mg}, \mathrm{Al}-\mathrm{Zn}-\mathrm{Mg}$ alloys, and $\mathrm{Al}$ : experiments, continuum, and atomistic modeling Corrosion 72 297-313

[33] Villars P and Calvert L 1985 Pearson's Handbook of Crystallographic Data for Intermetallic Phases (Materials Park, OH: ASM)

[34] Pozzo M, Carlini G, Rosei R and Alfè D 2007 Comparative study of water dissociation on $\mathrm{Rh}\left(\begin{array}{lll}1 & 1 & 1\end{array}\right)$ and $\mathrm{Ni}\left(\begin{array}{lll}1 & 1 & 1\end{array}\right)$ studied with first principles calculations J. Chem. Phys. 126164706

[35] Kittel C 1996 Introduction to Solid State Physics (New York: Wiley)

[36] Wu H H and Trinkle D R 2011 Direct diffusion through interpenetrating networks: oxygen in titanium Phys. Rev. Lett. 107045504 